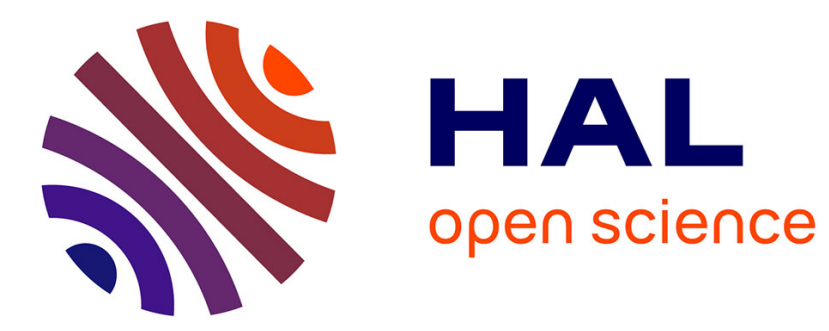

\title{
Effect of linker length on the spectroscopic properties of bacteriochlorin - 1,8-naphthalimide conjugates for fluorescence-guided photodynamic therapy
}

Pavel Panchenko, Marina Zakharko, Mikhail Grin, Andrey Mironov, Dmitriy Pritmov, Gediminas Jonusauskas, Yuri V Fedorov, Olga A Fedorova

\section{To cite this version:}

Pavel Panchenko, Marina Zakharko, Mikhail Grin, Andrey Mironov, Dmitriy Pritmov, et al.. Effect of linker length on the spectroscopic properties of bacteriochlorin - 1,8-naphthalimide conjugates for fluorescence-guided photodynamic therapy. Journal of Photochemistry and Photobiology A: Chemistry, 2019, 390, pp.112338. 10.1016/j.jphotochem.2019.112338 . hal-02423692

\section{HAL Id: hal-02423692 \\ https://hal.science/hal-02423692}

Submitted on 30 Dec 2020

HAL is a multi-disciplinary open access archive for the deposit and dissemination of scientific research documents, whether they are published or not. The documents may come from teaching and research institutions in France or abroad, or from public or private research centers.
L'archive ouverte pluridisciplinaire HAL, est destinée au dépôt et à la diffusion de documents scientifiques de niveau recherche, publiés ou non, émanant des établissements d'enseignement et de recherche français ou étrangers, des laboratoires publics ou privés. 


\title{
Effect of linker length on the spectroscopic properties of bacteriochlorin - 1, 8-naphthalimide conjugates for fluorescence-guided photodynamic therapy
}

\author{
Pavel A. Panchenko, a, ,*, Marina A. Zakharko ${ }^{a}$, Mikhail A. Grin ${ }^{c}$, Andrey F. Mironov ${ }^{c}$, \\ Dmitriy A. Pritmov ${ }^{c}$, Gediminas Jonusauskas ${ }^{\mathrm{d}}$, Yuri V. Fedorova ${ }^{\mathrm{a}}$, Olga A. Fedorova ${ }^{\mathrm{a}, \mathrm{b}}$ \\ ${ }^{a}$ A.N. Nesmeyanov Institute of Organoelement Compounds of Russian Academy of Sciences (INEOS RAS), 119991, Vavilova str. 28, Moscow, Russia \\ ${ }^{\mathrm{b}}$ Dmitry Mendeleev University of Chemical Technology of Russia, 125047, Miusskaya sqr. 9, Moscow, Russia \\ ${ }^{c}$ M.V. Lomonosov Institute of Fine Chemical Technologies, Russian Technological University (MIREA), 119571, Prospect Vernadskogo, 86, Moscow, Russia \\ ${ }^{\mathrm{d}}$ Laboratoire Ondes et Matière d'Aquitaine (LOMA), UMR CNRS 5798, Bordeaux University, 33405, 351 Cours de la Libération, Talence, France
}

\begin{abstract}
A B S T R A C T
Conjugation of 4-pyrazolinyl-1,8-naphthalimide and propargyl-15 $15^{2}, 17^{3}$-dimethoxy-13 $13^{1}$-amide of bacteriochlorin $e$ by the oligo(ethylene glycol) linker resulted in the dual function system comprising the modalities of a photosensitizer and a fluorescence imaging agent. In comparison with the conjugate of the same structure but containing the shorter linker, the system demonstrated the higher emission intensity originating from the naphthalimide unit, which could be explained by the decrease in resonance energy transfer efficiency between the chromophores. It has been shown that the ability of bacteriochlorin to generate singlet oxygen remains at the same level when this fragment is introduced in the conjugate structure. 4-Pyrazolinyl-1,8-naphthalimide group in the studied compounds was found to easily undergo photooxidation yielding highly emissive 4-pyrazolyl-1,8naphthalimide derivatives.
\end{abstract}

\section{Introduction}

Photodynamic therapy (PDT) is a relatively novel and minimally invasive treatment that can exert a selective cytotoxic activity towards malignant cells [1,2]. The method includes three necessary elements: a drug called photosensitizer (PS), light and molecular oxygen presenting in a solubilized form in cells and tissues. When irradiated with a specific wavelength of light, PS is first transformed to $S_{1}$ state. Subsequent intersystem crossing produces the triplets ${ }^{3} \mathrm{PS}^{*}$, which are capable to interact with ${ }^{3} \mathrm{O}_{2}$ upon collisions resulting in the production of cytotoxic singlet oxygen $\left({ }^{1} \mathrm{O}_{2}\right)$ and free radicals. This causes cell death via apoptosis or necrosis.

Derivatives of porphyrin, chlorin and bacteriochlorin are known to be one of the most commonly used types of photosensitizers for PDT. A significant advantage of these compounds is due to their ability to accumulate in tumors and to sensitize the formation of ${ }^{1} \mathrm{O}_{2}$ and other reactive oxygen species (ROS) with a high quantum yield. Many of these compounds have reached different stages of clinical or preclinical trials [3-7]. In addition to therapy, the difference in the concentration of PS in malignant and normal tissue can be the basis for fluorescence diagnostics. Fluorescence imaging methods are low-cost and non- invasive. In the meantime, they can provide an accurate estimations concerning the tumor size and its localization [8,9]. Unfortunately, porphyrin-based photosensitizers have very small Stokes shifts, which significantly limits their application in fluorescence imaging. Furthermore, excitation of PS during the diagnostic mode would obviously case related phototoxicity effects with respect to normal tissues.

To enhance the imaging capability of the PDT agents, a new approach, which consists in combining a fragment of a photosensitizer with a fragment of a fluorescent dye in one molecule, has been proposed [10-12]. Thus, it is expected that the excitation of the fluorophore would give a strong emission signal, which can be used to monitor the response of the tumor to treatment. Further excitation with a different wavelength of light corresponding to the absorption maximum of PS would lead to the formation of ROS responsible for cancer cell damage.

Recently, we have prepared conjugates of propargyl- $15^{2}, 17^{3}$-dimethoxy-13 $3^{1}$-amide of bacteriochlorin $e$ (BChl, Scheme 1 ) and $N$-aminobacteriopurpurinimide with 1,8-naphthalimide derivatives [13,14]. This type of fluorescent dyes exhibited high Stockes shifts, good photostability and imaging capability in vitro [14-16]. However, the fluorescence of naphthalimide core in the studied conjugates was found

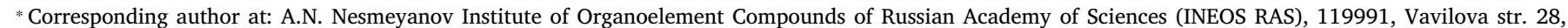
Moscow, Russia.

E-mail address: pavel@ineos.ac.ru (P.A. Panchenko). 


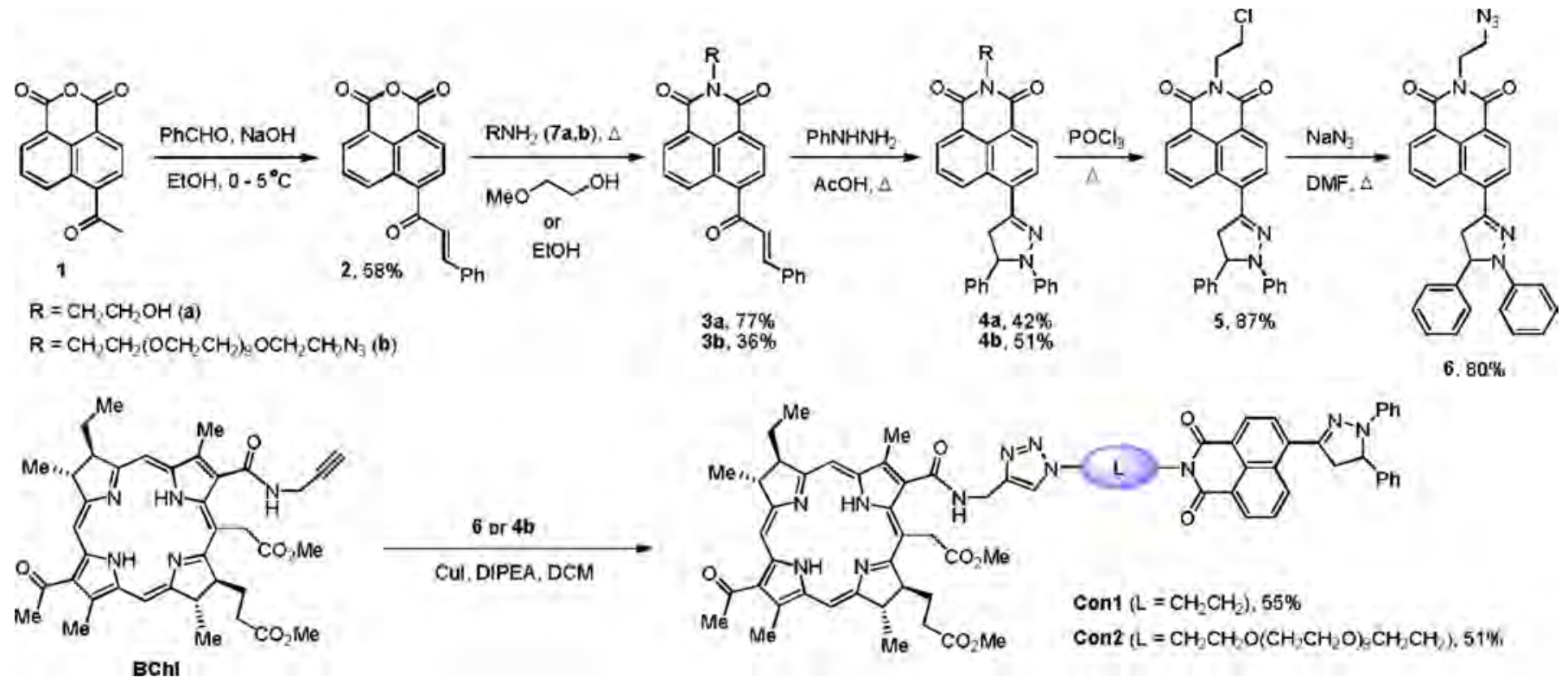

Scheme 1. Synthesis of Con1 and Con2.

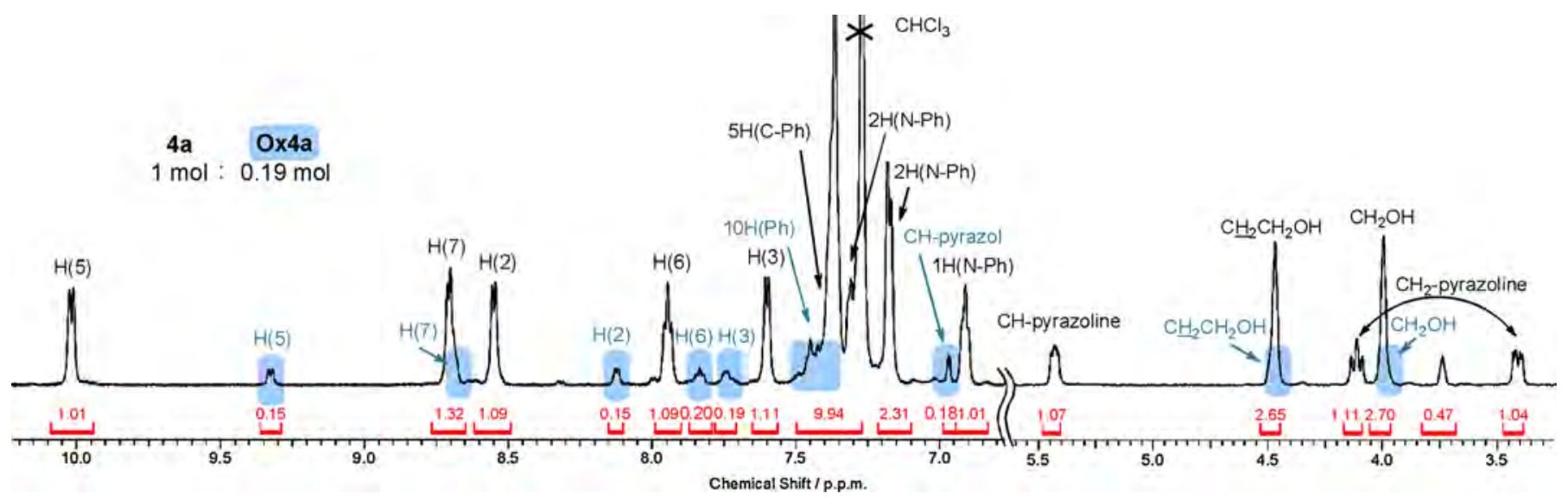

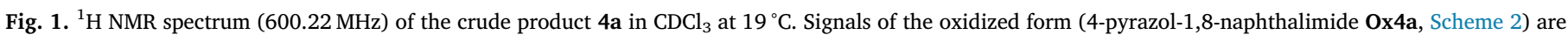
marked with blue color. Numbering of carbon atoms in the naphthalimide fragment of $\mathbf{4 a}$ and $\mathbf{O x} \mathbf{4} \mathbf{a}$ is shown in Scheme 2 .

to be completely quenched due to resonance energy transfer (RET) to the acceptor photosensitizer fragment. Thus, the imaging was only possible after the high power irradiation, which caused photodestruction of the bacteriochlorin chromophore [14].

It is well-known that the resonance energy transfer efficiency $\left(\Phi_{\mathrm{RET}}\right)$ in a bichromophoric system depends on the distance between the photoactive units ( $r$ ); namely, $\Phi_{\mathrm{RET}}$ is inversely proportional to $r^{6}$ if the Förster mechanism of RET (or FRET) is in operation [17]. In the present paper, we aimed to use the above-mentioned feature of FRET to improve the fluorescent characteristics of conjugates. For this purpose, two novel conjugates Con1 and Con2 (Scheme 1) combining 4-(1,5diphenyl- $\Delta^{2}$-pyrazolin-3-yl)-1,8-naphthalimide and bacteriochlorin $e$ units were obtained. The conjugates Con 1 and Con2 are different in the length of the linker group L connecting the fluorophore and PS moieties so that their energy transfer characterisctics might be also different. Furthermore, oligo(ethylene glycol) linker in Con2 could make the whole molecule rather amphiphilic; it could self-assemble into micelles with a suitable size not only for long body circulation but also for in vivo accumulation in the tumor area [18].

\section{Results and discussion}

\subsection{Synthesis of the compounds}

Conjugates Con 1 and Con2 were synthesized by the cycloaddition click reaction of the bacteriochlorin $e$ derivative $\mathbf{B C h l}$ and modified 4(1,5-diphenyl- $\Delta^{2}$-pyrazolin-3-yl)-1,8-naphthalimides 6 and $4 \mathrm{~b}$ bearing azide groups in the $N$-imide substituent (Scheme 1). Compound BChl was afforded from the methyl ester of bacteriopheophorbide $a$ and propargylamine as described earlier [14]. As a source of the former, biomass Rhodobacter capsulatus was used $[19,20]$. For the preparation of pyrazolinyl-1,8-naphthalimides $4 \mathrm{~b}$ and 6 , condensation of 4-acetylnaphthalic anhydride 1 with benzaldehyde was carried out. Then, the resulting product 2 was imidated with amines $7 \mathbf{a}$ and $7 \mathbf{b}$ to yield 4 cinnamoyl-1,8-naphthalimides $\mathbf{3} \mathbf{a}$ and $\mathbf{3} \mathbf{b}$, which were further subjected to condensation with phenylhydrazine. Regarding compound $\mathbf{6}$, introduction of the azide group was performed after the formation of pyrazolinyl ring via subsequent treatment of $4 \mathbf{a}$ with $\mathrm{POCl}_{3}$ and $\mathrm{NaN}_{3}$. This, however, was not the case for the compound $4 b$, which was 


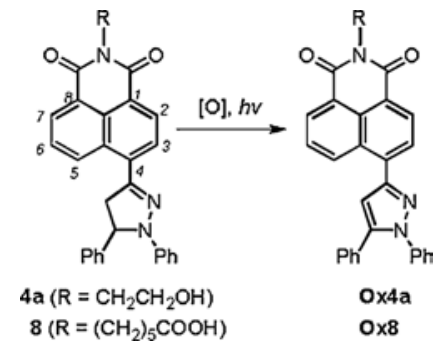

Scheme 2. Photooxidation of pyrazolinyl group.

prepared using commercially available oligo(ethylene glycol) derivative $\mathbf{7 b}$ containing the terminal azido group.

An interesting observation was found in the ${ }^{1} \mathrm{H}$ NMR spectra of the isolated products 4a, 5, 6, Con1 and Con2. Structure elucidations revealed that the signals of the naphthalimide fragment are split into two sets with the intensity ratio of about $1: 0.12 \div 0.40$. As an example, Fig. 1 shows the ${ }^{1} \mathrm{H}$ NMR spectrum of $4 \mathbf{a}$ in $\mathrm{CDCl}_{3}$ (see Supplementary information for the NMR spectra of other compounds). At the same time, EI mass spectra of $\mathbf{4 a}$ and $\mathbf{5}$ showed clear peaks of $[\mathrm{M}-2]^{+}$. species (Fig.S27,S29). Aiming to rationalize these results, we supposed that five-membered heterocyclic ring at C-4 position of the naphthalimide fragment could be oxidized to form 4-pyrazolyl group (Scheme 2). Such oxidation has been previously found for 1,3-diaryl-

and 1,3,5-triaryl-2-pyrazolines [21-24] as well as for pyrazolinesubstituted coumarins [25-27] either upon irradiation in the presence of oxygen or even when irradiation in degassed solution is applied. The possible mechanism involves sensitization of singlet oxygen formation by a dye molecule or, alternatively, the electron transfer from the photoexcited chromophore to the solvent (especially for solvents like $\mathrm{CCl}_{4}$ or $\mathrm{C}_{2} \mathrm{Cl}_{6}$ ). Unfortunately, we were not able to isolate pure pyrazolinyl derivatives in all cases, and the mixtures of the non-oxidized with some amount of the oxidized form (usually $11-29 \mathrm{~mol}$. \% as revealed from ${ }^{1} \mathrm{H}$ NMR spectra) were used for the subsequent synthetic steps and photophysical studies.

Possibility of the photooxidation was evaluated using the previously obtained model compound 8 (Scheme 2) [28] in which the presence of polar $\mathrm{COOH}$ group on the one hand provides good solubility and on the other makes the substituent at the $\mathrm{N}$-imide nitrogen of the napthalimide ring be resistible to oxidizing agents (in contrast to $\mathrm{CH}_{2} \mathrm{CH}_{2} \mathrm{OH}$ group in 4a). First, a solution of $8(10 \mu \mathrm{M})$ in $\mathrm{CHCl}_{3}$ was irradiated by the full light of high pressure $\mathrm{Hg}$ vapour lamp $(120 \mathrm{~W})$ during $90 \mathrm{~s}$, and the absorption spectra were recorded every 3,5 or $10 \mathrm{~s}$. It was found that irradiation caused bleaching of the absorption band of the 4-pyrazolinyl-1,8-naphthalimide ( $\lambda_{\max }^{\mathrm{fl}} 495 \mathrm{~nm}$ ) and a concomitant appearance of the new band at $365 \mathrm{~nm}$ with the isosbestic point at $409 \mathrm{~nm}$ (Fig.S33). The oxidation product $\mathbf{0 x 8}$ was isolated as a precipitate after the irradiation was ceased (see Supplementary information for characterization data of $\mathbf{0 x 8}$ ). The ${ }^{1} \mathrm{H}$ NMR spectrum of compound 8 $(7.6 \mathrm{mM})$ in $\mathrm{CDCl}_{3}$ under irradiation at the same conditions demonstrated the growth of the intensities for proton resonances of the oxidized form (0x8), the signals of pyrazolinylnaphthalimide 8 being completely disappeared after $40 \mathrm{~min}$ of exposure to the UV light (Fig.S34). Yet, compound $\mathbf{0 x 8}$ was also obtained by the oxidation of $\mathbf{8}$ with $\mathrm{K}_{2} \mathrm{Cr}_{2} \mathrm{O}_{7}$ in $\mathrm{AcOH}$ (see Supplementary Information).

\subsection{Photophysical characterization of dyes}

Steady-state absorption and emission properties of conjugates Con 1 and Con2 as well as monochromophoric derivatives $\mathbf{B C h l}$ and $\mathbf{4 a}$ are shown in Fig. 2 and Table 1. As it can be seen from Fig. 2a,c, absorption spectra of Con1, Con2 and equimolar mixture of BChl and 4a are very similar, each showing the presence of four bands of almost the same intensity at around 355, 480, 515 and $750 \mathrm{~nm}$ corresponding to electronic transitions in naphthalimide $(480 \mathrm{~nm})$ and bacteriochlorin (355,
515 and $750 \mathrm{~nm}$ ) moieties. Thus, the lack of $\pi, \pi$-interaction between the covalently linked chromophores could be suggested in the ground state.

The long wavelength absorption band of compound 4a with maximum at $482 \mathrm{~nm}$ can be attributed to intramolecular charge transfer (ICT) from the pyrazolinyl fragment to carbonyl groups of dicarboxyimide moiety [29]. Due to rather strong ICT nature of the lowest energy transition such type of chromophores could be excited by twophoton absorption with an IR laser. Indeed, in our recent paper [28], we have shown that fluorescence output from the carboxylic derivative 8 (the analogue of 4a) can be obtained in vitro by irradiation with $880 \mathrm{~nm}$ light which perfectly fits the phototherapeutic window $(600-1200 \mathrm{~nm})$.

Emission spectra of all compounds presented in Table 1 (except BChl) were recorded using $470 \mathrm{~nm}$ excitation wavelength $\left(\lambda_{\text {ex }}\right)$. Considering the changes in the UV/Vis absorption spectrum of compound $\mathbf{8}$ induced by the irradiation (Fig.S33), one can suppose that such excitation light would not be absorbed by the oxidized form and, therefore, all the collected spectra would arise from the direct excitation of the 4-pyrazolinyl-1,8-naphthalimide chromophore. To supply this assumption, we have measured the fluorescence emission spectra of the crude product $4 \mathrm{a}$ at $\lambda_{\mathrm{ex}} 360$ and $470 \mathrm{~nm}$ (Fig.S36b, Fig.1b). Indeed, the spectrum at $\lambda_{\text {ex }} 360 \mathrm{~nm}$ showed the intense peak at $475 \mathrm{~nm}$, whereas the long wavelength excitation produced only one band with $\lambda_{\max }^{\mathrm{fl}}$ $651 \mathrm{~nm}$. Excitation spectra obtained at 480 and $680 \mathrm{~nm}$ clearly show different emission peaks centered at 367 and $480 \mathrm{~nm}$ respectively (Fig.S36a). These observations are consistent with the highly emissive character of the oxidized form ${ }^{1}$ that absorbs at around $350-370 \mathrm{~nm}$ fluoresces in the blue region $\left(\lambda_{\max }^{\mathrm{fl}} 475 \mathrm{~nm}\right)$.

Fluorescence maxima of BChl appears at $755 \mathrm{~nm}$ (Fig. 2b). When the solution containing the mixture of equal amounts of BChl and 4a was excited at $470 \mathrm{~nm}$, we observed the broad-band fluorescence which was very similar to that of $\mathbf{4 a}$. This can be understood by the fact that the most part of the $470 \mathrm{~nm}$ light is absorbed by the naphthalimide fragment and only minor direct excitation of the bacteroichlorin unit occurs leading to the low intensity long wavelength shoulder in the spectrum (Fig. 2d). The spectrum of Con 1 containing the short linker between the chromophores was found to be completely different (Fig. 2d). Following the excitation at $470 \mathrm{~nm}$, this compound exhibited the characteristic peak of the bacteriochlorin and no emission signal from the naphthalimide was detected. Such result could be consistent with the possible resonance energy transfer from the fluorophore to the PS in the system.

To verify our assumption if resonance energy transfer is a reason of quenching of naphthalimide fluorescence in Con1, we calculated $\Phi_{\mathrm{RET}}$ using the Förster theory [17]. In this treatment, $\Phi_{\mathrm{RET}}$ can be expressed by Eq. (1):

$\Phi_{\mathrm{RET}}=\frac{R_{0}^{6}}{R_{0}^{6}+r^{6}}$

where $r$ is the distance between the donor and the acceptor and $R_{0}$ is the critical Förster radius at which $50 \%$ of the excited donors deactivate through the RET pathway (i.e. $\Phi_{\mathrm{RET}}=0.5$ ). The value $r$ was found from the optimized ground state geometry of Con1 (Fig. 3, $r=11.3 \AA$ ), and $R_{0}$ was calculated according to the Eq. (2):

$\mathrm{R}_{0}^{6}=\frac{9000 \ln 10 \kappa^{2} \varphi_{D, 0}^{\mathrm{fl}}}{128 \pi^{2} \mathrm{~N}_{\mathrm{A}} \mathrm{n}^{4}} \int_{0}^{\infty} \mathrm{F}_{\mathrm{D}}(\lambda) \varepsilon_{\mathrm{A}}(\lambda) \lambda^{4} \mathrm{~d} \lambda$

where $\kappa^{2}$ is an orientation factor which depends on the mutual disposition of the donor and acceptor transition dipole moments, $\varphi_{D, 0}^{\mathrm{fl}}$ is the fluorescence quantum yield of the donor in the absence of the acceptor,

\footnotetext{
${ }^{1}$ The assumption about highly emissive character of the oxidized forms of the pyrazolinyl-1,8-naphthalimide derivatives is based on the calculation of the

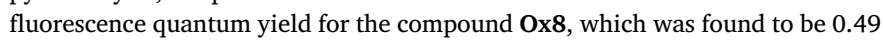
in $\mathrm{MeCN}$
} 

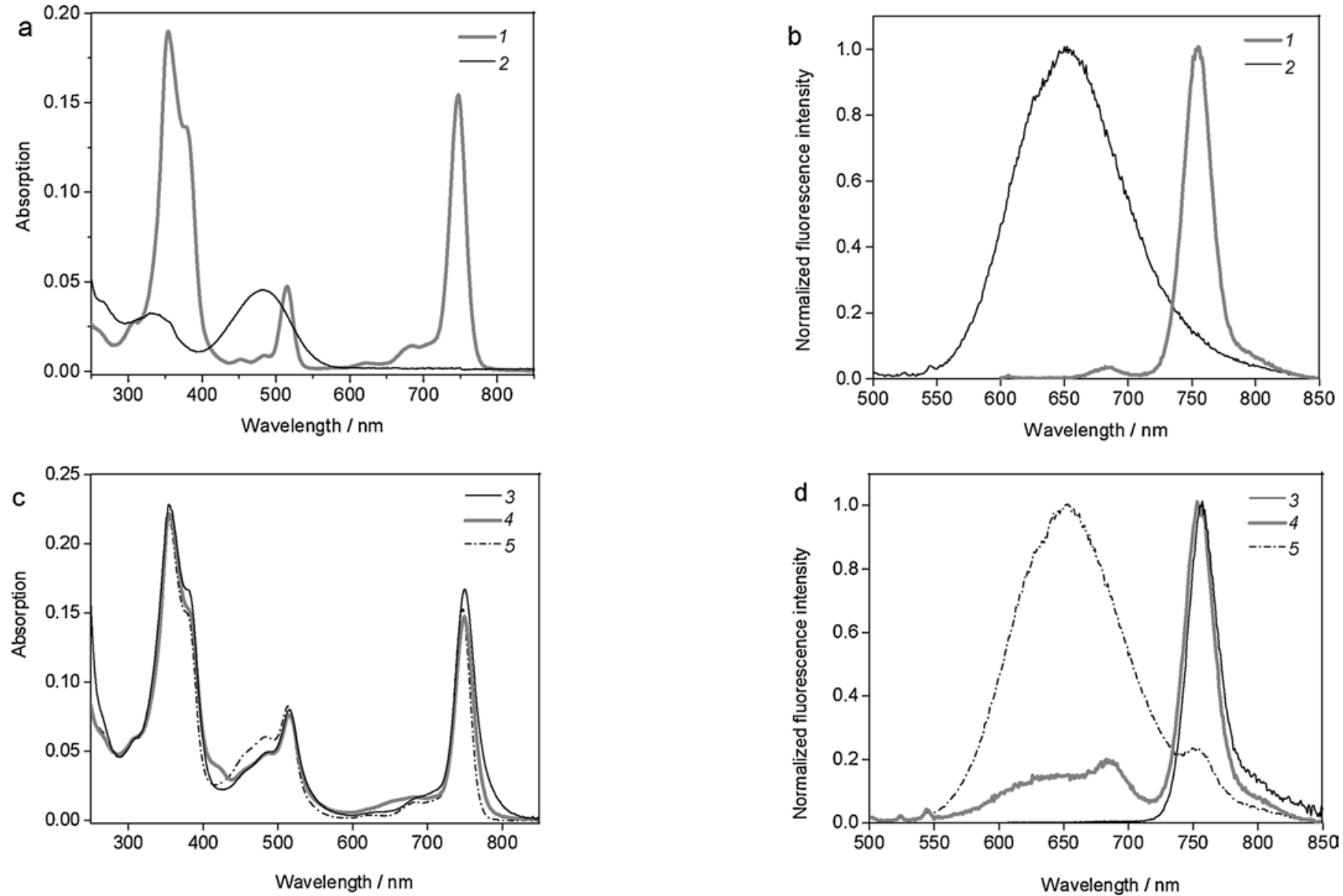

Fig. 2. UV/Vis absorption (a,c) and fluorescence emission (b, d) spectra of compounds BChl (1), 4a (2), Con1 (3), Con2 (4) and equimolar mixture of BChl and 4a (5) in acetonitrile. Excitation wavelength is $470 \mathrm{~nm}$ for $4 \mathrm{a}$, Con1, Con2, Mix and $515 \mathrm{~nm}$ for prop. Concentration of all compounds $-5.0 \cdot 10^{-6} \mathrm{M}$.

Table 1

. Photophysical characteristics ${ }^{\mathrm{a}}$ of compounds $4 \mathrm{a}, \mathbf{B C h}$, Con1 and Con2 in acetonitrile. ${ }^{\mathrm{b}}$.

\begin{tabular}{|c|c|c|c|c|c|c|c|c|c|}
\hline & $\lambda_{\max }^{\mathrm{abs}} / \mathrm{nm}$ & $\lambda_{\max }^{\mathrm{fl}}\left(\lambda_{\mathrm{ex}}\right) / \mathrm{nm}$ & $\varphi^{\mathrm{fl}}$ & $\tau_{\mathrm{D}}\left(\right.$ or $\left.\tau_{\mathrm{D}, 0}\right) / \mathrm{ns}$ & $R_{0} / \AA$ & \multicolumn{3}{|c|}{ Resonance energy transfer efficiency $\Phi_{\text {RET }}$} & $\Phi_{\Delta}\left(\lambda_{\mathrm{ex}} / \mathrm{nm}\right)$ \\
\hline $4 a$ & 482 & $651(470)$ & 0.071 & 0.93 & - & - & - & - & - \\
\hline Con1 & $355,515,749$ & $757(470)$ & 0.028 & not determined & 30.8 & 0.998 & not determined & not determined & $0.67(510)$ \\
\hline Con2 & $355,515,749$ & $753(470)$ & 0.021 & 0.065 & 30.8 & 0.065 & 0.929 & 0.930 & $0.83(510)$ \\
\hline
\end{tabular}

${ }^{\mathrm{a}} \lambda_{\max }^{\mathrm{abs}}$ and $\lambda_{\max }^{\mathrm{fl}}$ denote the maxima positions of absorbtion and emission bands respectively; $\lambda_{\mathrm{ex}}-$ excitation wavelength; $\varphi^{\mathrm{fl}}-$ fluorescence quantum yield; $\tau_{\mathrm{D}}$ and $\tau_{\mathrm{D}, 0}-$ excited state lifetime of the naphthalimide chromophore in the presence and absence of energy acceptor respectively; $R_{0}-$ critical Förster distance; $\Phi_{\Delta}-$ quantum yield of singlet oxygen generation.

${ }^{\mathrm{b}} \Phi_{\Delta}$ values were measured in acetone.

$n$ is the refractive index of the solvent, $N_{\mathrm{A}}$ is Avogadro constant and the integral corresponds to the extent of overlap between the normalized emission spectrum of the donor $\left(F_{\mathrm{D}}(\lambda)\right)$ and the absorption spectrum of the acceptor $\left(\varepsilon_{\mathrm{A}}(\lambda)\right.$, see Fig.S37a for the graphical representation of this overlap). Taking into account that $\kappa^{2}=2 / 3$ (for a random orientation) and $\varphi_{D, 0}^{\mathrm{fl}}=0.071$ (the quantum yield of $4 \mathrm{a}$ in acetonitrile), we found the RET efficiency $\Phi_{\text {RET }}$ to be as high as 0.998 . Exactly the same value of $\Phi_{\text {RET }}$ was found both from theoretical calculations (0.995) and the analysis of the transient absorption spectra (0.999) for the previously reported conjugate of similar structure [14], in which 4-N,N-dimethylaminostyryl group is presented instead of 1,5-diphenyl- $\Delta^{2}$-pyrazolin-3yl one.

In contrast to Con1, excitation of Con2 with $470 \mathrm{~nm}$ light produced the broad emission band at around $650 \mathrm{~nm}$ attributed to the fluorescence of the naphthalimide unit (Fig. 2d), thus showing that the RET is less effective. Yet, the relaxation of naphthalimide excited state in Con2 was long enough ( $\tau_{D}=65 \mathrm{ps}$, Table 1 ), so we could measure it using our time-resolved fluorescence setup (see the description in [16]). Comparison of the obtained lifetime $\tau_{\mathrm{D}}$ with that of the individual donor chromophore $\tau_{\mathrm{D}, 0}$ taken apart of the energy acceptor $(0.93 \mathrm{~ns}$, the

lifetime of 4a, see Table 1) allowed to estimate the experimental value of RET efficiency (Eq. (3)) $)^{2}$. As found from Eq. (3), $\Phi_{\text {RET }}$ for Con2 was 0.930 , which is obviously lower than $\Phi_{\mathrm{RET}}$ in the case of Con1. Essentially the same $\Phi_{\text {RET }}$ value (0.929) was also obtained by the Eq. (4)

\footnotetext{
${ }^{2}$ In the case of Con1, calculation of $\Phi_{\mathrm{RET}}$ by the Equation [3] was not done because the lifetime of the excited naphthalimide fragment appears to be lower compared to temporal resolution in the experiment.
} 

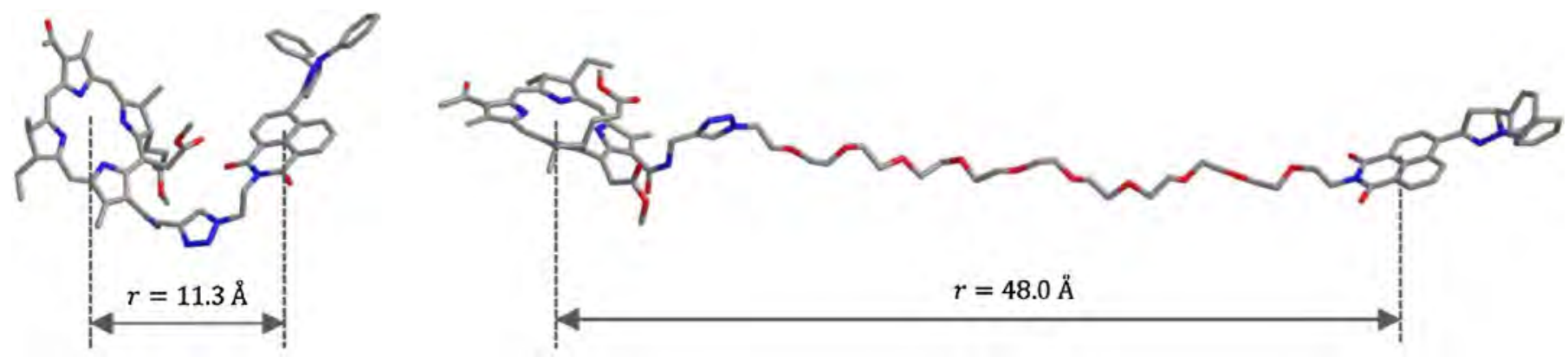

Fig. 3. Ground state geometry of Con1 (left) and Con2 (right) calculated using PM6 method. Hydrogen atoms are omitted for the sake of simplicity.

where the steady-state emission intensities of the energy donor in the presence $\left(I_{\mathrm{D}}\right)$ and absence $\left(I_{\mathrm{D}, 0}\right)$ of the acceptor are used instead of corresponding lifetimes (see Fig.S37b for the non-normalized fluorescence spectra of Con2 and equimolar mixture of BChl and 4a showing the $I_{\mathrm{D}}$ and $I_{\mathrm{D}, 0}$ values). ${ }^{3}$

$\Phi_{\mathrm{RET}}=1-\frac{\tau_{\mathrm{D}}}{\tau_{\mathrm{D}, 0}}$

$\Phi_{\mathrm{RET}}=1-\frac{I_{\mathrm{D}}}{I_{\mathrm{D}, 0}}$

Further analysis revealed that the presented experimental estimations of $\Phi_{R E T}$ in the case of Con2 did not match the result of the theoretical calculation by the Förster model like in the case of Con1. According to Fig. 3, the distance between naphthalimide and bacteriochlorin units in Con2 molecule could be as high as $48.0 \AA$. This in turn should result in only $6.5 \%$ of RET efficiency in the system, provided that the critical distance $R_{0}$ in the Eq. (1) is the same. The higher experimental value of $\Phi_{\mathrm{RET}}(\sim 93 \%)$ can be explained by a rather high sterical flexibility of the oligo(ethylene glycol) linker, which could lead to a situation when the majority of Con2 molecules adopt the conformations where the distance between the naphthalimide and bacteriochlorin chromophores is lower than $R_{0}$ and, hence, an efficient RET could occur.

In order to test the applicability of Con1 and Con2 as bioimaging agents, we also measured their absorption and fluorescence spectra in rabbit blood serum (Fig.S38). Such a model medium makes it possible to roughly estimate how the complex biological microenvironment in which the fluorescent probe is immersed may affect its optical characteristics. As for Con2, there is no significant difference in the relative fluorescence intensities of naphthalimide fragment and bacteriochlorin core upon going from the acetonitrile solution (Fig. 2d) to rabbit blood serum (Fig.S38d). However, an obvious increase in the fluorescence intensity of naphthalimide unit can be deduced from the same comparison in the case of Con1. This observation could be a result of conformational changes in the Con 1 structure upon its possible interactions with serum components, which might cause a decrease in the orientation factor $\kappa^{2}$ value (see Eq.s (1) and (2)) thereby reducing the RET efficiency. In general, the data presented allow us to conclude that both conjugates will not impair their fluorescent characteristics when replacing an organic solvent with an aqueous biological medium.

Generation of ${ }^{1} \mathrm{O}_{2}$ was investigated using 1,3-diphenylisobenzofuran (DPBF) as a singlet oxygen trap. The reaction between DPBF and ${ }^{1} \mathrm{O}_{2}$ results in the formation of endoperoxides exhibiting the absorption in the more short wavelength region compared to DPBF [30,31]. Thus, formation of singlet oxygen leads to a decrease in absorption at the main maximum of DPBF (414 nm). Rapid photobleaching of DPBF was detected in solutions of Con1, Con2 and free bacteriochlorin BCh1

\footnotetext{
${ }^{3}$ Similar estimation of $\Phi_{\mathrm{RET}}$ for Con1 using Equation [4] is impossible due to the lack of emission signal at $650 \mathrm{~nm}$ in the spectrum of Con1 (Fig. 2d) corresponding to donor chromophore fluorescence.
}

(Fig.S38a-c). In the solution of the free naphthalimide dye 4a, photobleaching of the trap was not found (Fig.S38d). Quantum yields of singlet oxygen sensitization $\left(\Phi_{\Delta}\right)$ of Con1, Con2 and BChl under the excitation at $510 \mathrm{~nm}$ were found to be $0.67,0.83$ and 0.79 respectively (Table 1). Such close values indicate that photosensitizing activity of the bacteriochlorin is not affected by the presence of naphthalimide fragment in Con1 and Con2.

\section{Conclusion}

In summary, two novel conjugates bearing bacteriochlorin photosensitizer and 4-pyrazolinyl-1,8-naphthalimide fluorophore have been synthesized. Regardless of the linker length, the presence of the naphthalimide fragment does not decrease the ability of PS core to generate ${ }^{1} \mathrm{O}_{2}$. Using the steady-state and time-resolved spectroscopic data, we have demonstrated that introduction of the flexible oligo(ethylene glycol) linker between the chromophores suppress the RET to some extent and, thus, leads to an increase in the emission intensity of the naphthalimide fragment. Thus, the studied conjugate Con2 could be considered as a promising candidate for the application as photosensitizing and imaging agent.

\section{Experimental part}

4-Acetylnaphthalic anhydride $\mathbf{1}$ was obtained from acenaphthene according to the known method [32]. Propargyl-152,173-dimethoxy131-amide of bacteriochlorin e (BChl) was synthesized according previously published procedure [14]. All other reagents were purchased from commercial sources and were of the highest grade. Synthetic procedures used for the preparation of compounds 2 , 3a, 3b, 4a, 4b, 5, 6, Con1 and Con2 as well as characterization data are listed in Supplementary information.

The absorption spectra were taken on a Varian-Cary $5 \mathrm{G}$ spectrophotometer. The fluorescence quantum yield measurements were performed using a Varian-Cary $5 \mathrm{G}$ spectrophotometer and a FluoroLog-3221 spectrofluorimeter. Spectral measurements were carried out in airsaturated acetonitrile solutions (acetonitrile of spectrophotometric grade, water content $<0.005 \%$, Aldrich). All measured fluorescence spectra were corrected for the nonuniformity of detector spectral sensitivity. Fluorescence quantum yields were determined using Coumarin 481 in acetonitrile $\left(\varphi^{\mathrm{fl}}=0.08\right)$ [33] as a reference. The quantum yields of singlet oxygen $\left(\Phi_{\Delta}\right)$ were estimated in acetone by using tetraphenylporphyrin (TPP) as a reference compound $\left(\Phi_{\Delta}^{\mathrm{TPP}}=0.7\right)[30,31]$ and DPBF as a ${ }^{1} \mathrm{O}_{2}$ trap following the described method [14].

The three dimentional structure of Con 1 and Con 2 were built with MOPAC 2016 program package using PM6 semiempirical method [34]. The calculations were performed at optimized geometries, which reached gradient variations less than $0.01 \mathrm{kcal} / \mathrm{mol}$. The solvent effect was included in geometry optimizations following the «COnductorlike Screening Model»(COSMO) implemented in MOPAC. A dielectric constant of $\varepsilon=20$ and a refraction index of solvent $(n)$ such that $n^{2}=2$ were used. 


\section{CRediT authorship contribution statement}

Pavel A. Panchenko: Writing - original draft, Methodology, Formal analysis. Marina A. Zakharko: Investigation, Visualization. Mikhail A. Grin: Conceptualization. Andrey F. Mironov: Supervision. Dmitriy A. Pritmov: Investigation, Visualization. Gediminas Jonusauskas: Methodology, Investigation, Formal analysis. Yuri V. Fedorov: Methodology, Writing - review \& editing. Olga A. Fedorova: Conceptualization, Supervision.

\section{Declaration of Competing Interest}

There are no conflicts to declare

\section{Acknowledgements}

The work was supported by Russian Science Foundation (grant № 16-13-10226). Steady-state studies were performed with the financial support from Ministry of Science and Higher Education of the Russian Federation using the equipment of Center for molecular composition studies of INEOS RAS.

\section{References}

[1] J.P. Celli, B.Q. Spring, I. Rizvi, C.L. Evans, K.S. Samkoe, S. Verma, B.W. Pogue, T. Hasan, Imaging and photodynamic therapy: mechanisms, monitoring, and optimization, Chem. Rev. 110 (2010) 2795-2838, https://doi.org/10.1021/cr900300p.

[2] P. Agostinis, K. Berg, K.A. Cengel, T.H. Foster, A.W. Girotti, S.O. Gollnick, S.M. Hahn, M.R. Hamblin, A. Juzeniene, D. Kessel, M. Korbelik, J. Moan, P. Mroz, D. Nowis, J. Piette, B.C. Wilson, J. Golab, Photodynamic therapy of cancer: an update, CA Cancer J. Clin. 61 (2011) 250-281, https://doi.org/10.3322/caac. 20114.

[3] D.A. Belnier, W.R. Greco, G.M. Loewen, V. Nava, A.O. Oseroff, R.K. Pandey, T. Tsuchida, T.J. Dougherty, Population pharmacokinetics of the photodynamic agent 2-[1-Hexyloxyethyl]-2-devinyl Pyropheophorbide-a in cancer patients, Cancer Res. 63 (2003) 1806-1813.

[4] N.E. Martin, S.M. Hahn, Interstitial photodynamic therapy for prostate cancer: a developing modality, Photodiagn. Photodyn. Ther. 1 (2004) 123-136, https://doi. org/10.1016/S1572-1000(04)00037-7.

[5] Y. Chen, G. Li, R.K. Pandey, Synthesis of Bacteriochlorins and their potential utility in photodynamic therapy (PDT), Curr. Org. Chem. 8 (2004) 1105-1134, https:// doi.org/10.2174/1385272043370131.

[6] J.D. Miller, E.D. Baron, H. Scull, A. Hsia, J.C. Berlin, T. McCormick, V. Colussi, M.E. Kenny, K.D. Cooper, N.L. Olienick, Photodynamic therapy with the phthalocyanine photosensitizer Pc 4: the case experience with preclinical mechanistic and early clinicaltranslational studies, Toxicol. Appl. Pharmacol. 224 (2007) 290-299, https://doi.org/10.1016/j.taap.2007.01.025.

[7] R.A. Weersink, A. Bogaards, M. Gertner, S.R.H. Davidson, K. Zhang, G. Netchev, T. Trachterberg, B.C. Wilson, Techniques for delivery and monitoring of TOOKAD (WST09)-mediated photodynamic therapy of the prostate: clinical experience and practicalities, J. Photochem, J. Photochem. Photobiol. B, Biol. 79 (2005) 211-222, https://doi.org/10.1016/j.jphotobiol.2005.01.008.

[8] P.K. Pandey, N. James, Y. Chen, M.P. Dobhal, Cyanine dye based compounds for tumor imaging with and without photodynamic therapy, Top. Heterocycl. Chem. 14 (2008) 41-74

[9] H.R. Jager, M.N. Taylor, T. Theodossy, C. Hopper, MR imaging-guided interstitial photodynamic laser therapy for advanced head and neck tumors, Am. J. Neuroradiol. 26 (2005) 1193-1200

[10] M.P.A. Williams, M. Ethirajan, K. Ohkubo, P. Chen, P. Pera, J. Morgan, W.H. White III, M. Shibata, S. Fukuzumi, K.M. Kadish, R.K. Pandey, Synthesis, photophysical, electrochemical, tumor-imaging, and phototherapeutic properties of purpurinimide-N-substituted cyanine dyes joined with variable lengths of linkers, Bioconjugate Chem. 22 (2011) 2283-2295, https://doi.org/10.1021/bc200345p.

[11] N.S. James, T.Y. Ohulchanskyy, Y. Chen, P. Joshi, X. Zheng, L.N. Goswami, R.K. Pandey, Comparative tumor imaging and PDT efficacy of HPPH conjugated in the mono- and di-forms to various polymethine cyanine dyes: part -2 , Theranostis 3 (2013) 703-718, https://doi.org/10.7150/thno.5923.
[12] N.S. James, P. Joshi, T.Y. Ohulchanskyy, Y. Chen, W. Tabaczynski, F. Durrani, M. Shibata, R.K. Pandey, Photosensitizer (PS)-cyanine dye (CD) conjugates: impact of the linkers joining the PS and CD moieties and their orientation in tumor-uptake and photodynamic therapy (PDT), Eur. J. Med. Chem. 122 (2016) 770-785, https://doi.org/10.1016/j.ejmech.2016.06.045.

[13] P.A. Panchenko, A.N. Sergeeva, O.A. Fedorova, Yu.V. Fedorov, R.I. Reshetnikov, A.E. Schelkunova, M.A. Grin, A.F. Mironov, G. Jonusauskas, Spectroscopical study of bacteriopurpurinimide-naphthalimide conjugates for fluorescent diagnostics and photodynamic therapy, J. Photochem. Photobiol. B 133 (2014) 140-144, https:// doi.org/10.1016/j.jphotobiol.2014.03.008.

[14] P.A. Panchenko, M.A. Grin, O.A. Fedorova, M.A. Zakharko, D.A. Pritmov, A.F. Mironov, A.N. Arkhipova, Yu.V. Fedorov, G. Jonusauskas, R.I. Yakubovskaya, N.B. Morozova, A.A. Ignatova, A.V. Feofanov, A novel bacteriochlorin-styrylnaphthalimide conjugate for simultaneous photodynamic therapy and fluorescence imaging, Phys. Chem. Chem. Phys. 19 (2017) 30195-30206, https:// doi.org/10.1039/c7cp04449f.

[15] H.-H. Lin, Y.-Ch. Chan, J.-W. Chen, Ch.-Ch. Chang, Aggregation-induced emission enhancement characteristics of naphthalimide derivatives and their applications in cell imaging, J. Mater. Chem. 21 (2011) 3170-3177, https://doi.org/10.1039/ C0JM02942D.

[16] P.A. Panchenko, A.N. Arkhipova, O.A. Fedorova, Yu.V. Fedorov, M.A. Zakharko, D.E. Arkhipov, G. Jonusauskas, Controlling photophysics of styrylnaphthalimides through TICT, fluorescence and E,Z-photoisomerization interplay, Phys. Chem. Chem. Phys. 19 (2017) 1244-1256, https://doi.org/10.1039/C6CP07255K.

[17] J.R. Lakowicz, Principles of Fluorescence Spectroscopy, Springer, New York, USA, 2006.

[18] Z. Ruan, Y. Zhao, P. Yuan, L. Liu, Y. Wang, L. Yan, PEG conjugated BODIPY-Br $\mathrm{Br}_{2}$ as macro-photosensitizer for efficient imaging-guided photodynamic therapy, J. Mater. Chm. B 6 (2018) 753-762, https://doi.org/10.1039/C7TB02924A.

[19] A. Scherz, Y. Solomon, A. Brandis and H. Scheer, Pat., WO00/33833, 2000.

[20] G. Zheng, B. Chance and J. D. Glickson, Pat.,WO2006/073419 A2, 2006.

[21] N.A. Evans, Dye-sensitized photooxidation of some substituted 1,3-Diphenyl-2pyrazolines, Aust. J. Chem. 28 (1975) 433-437, https://doi.org/10.1071/ CH9750433.

[22] M. Mella, M. Fagnoni, G. Viscardi, P. Savarino, F. Elisei, A. Albini, On the photochemical behavior of some diarylpyrazolines, J. Photochem. Photobiol. A Chem. 108 (1997) 143-148, https://doi.org/10.1016/S1010-6030(97)00097-X.

[23] P. Ye, D.-H. Wang, B. Chen, Q.-Y. Meng, C.-H. Tung, L.-Z. Wu, Visible light catalyzed aromatization of 1,3,5-triaryl-2-pyrazolines by platinum(II) polypyridyl complex under oxidant-free condition, Sci. China Chem. 59 (2016) 175-179, https://doi.org/10.1007/s1142.

[24] V.F. Traven, I.V. Ivanov, S.M. Dolotov, A.N. Semakin, D.A. Cheptsov, Z.Z. Mamirgova, Efficient photooxidation of aryl(hetaryl)pyrazolines by benzoquinone, Photochem. Photobiol. 95 (2019) 924-930, https://doi.org/10.1111/php. 13082

[25] V.F. Traven, I.V. Ivanov, A.S. Pavlov, A.V. Manaev, I.V. Voevodina, V.A. Barachevskii, Quantitative photooxidation of 4-hydroxy-3-pyrazolinylcoumarins to pyrazolyl derivatives, Mendeleev Commun. 17 (2007) 345-346, https://doi.org/10.1016/j.mencom.2007.11.016.

[26] V.F. Traven, I.V. Ivanov, New reaction of photoaromatization of aryl and hetarylpyrazolines, Russ. Chem. Bull. 57 (2008) 1063-1069, https://doi.org/10.1007/ s11172-008-0135-3.

[27] V.F. Traven, S.M. Dolotov, I.V. Ivanov, Activation of fluorescence of lactone form of rhodamine dyes by photodehydrogenation of aryl(hetaryl)pyrazolines, Russ. Chem. Bull. 65 (2016) 735-740, https://doi.org/10.1007/s11172-016-1365-4.

[28] L. Francés-Soriano, M.A. Zakharko, M. González-Béja, P.A. Panchenko, V. HerranzPérez, D.A. Pritmov, M.A. Grin, A.F. Mironov, J.M. García-Verdugo, O.A. Fedorova, J. Pérez-Prieto, A nanohybrid for photodynamic therapy and fluorescence imaging tracking without therapy, Chem. Mater. 30 (2018) 3677-3682, https://doi.org/10 1021/acs.chemmater.8b00276.

[29] A.N. Sergeeva, P.A. Panchenko, Yu.V. Fedorov, O.A. Fedorova, Synthesis and sensor properties of crown-containing derivatives of 4-(1,5-Diphenyl- $\Delta^{2}$-Pyrazolin-3-yl)1,8-Naphthalimide, Protec. Met. Phys. Chem. Surf. 48 (2012) 524-533, https://doi. org/10.1134/S2070205112050103.

[30] A.A. Krasnovsky Jr., A.S. Kozlova, Ya.V. Roumbal, Photochemical investigation of the IR absorption bands of molecular oxygenin organic and aqueous environment, Photochem. Photobiol. Sci. 11 (2012) 988-997, https://doi.org/10.1039/ c2pp05350k.

[31] F. Wilkinson, W.P. Helman, A.B. Ross, Quantum yields for the photosensitized formation of the lowest electronically excited singlet state of molecular oxygen in solution, J. Phys. Chem. Ref. Data 22 (1993) 113-262, https://doi.org/10.1063/1 555934.

[32] W. Zhu, R. Yao, H. Tian, Synthesis of novel electro-transporting emitting compounds, Dyes Pigm. 54 (2002) 147-154, https://doi.org/10.1016/S0143-7208(02) 00039-6.

[33] S. Nad, M. Kumbhakar, H. Pal, Photophysical properties of Coumarin-152 and Coumarin-481 dyes: unusual behavior in Nonpolar and in higher polarity solvents, J. Phys. Chem. A 107 (2003) 4808-4816, https://doi.org/10.1021/jp021543t.

[34] J.J.P. Stewart, Optimization of parameters for semiempirical methods V: modification of NDDO approximations and application to 70 elements, J. Mol. Model. 13 (2007) 1173-1213, https://doi.org/10.1007/s00894-007-0233-4. 